\title{
Gold Nanoparticles Embedded on the Surface of Polyvinyl Alcohol Layer
}

\author{
Sasha Md. Nasir and Hadi Nur* \\ Ibnu Sina Institute for Fundamental Science Studies, Universiti Teknologi Malaysia, 81310 UTM Skudai, Johor, Malaysia. \\ *To whom correspondence should be addressed. E-mail: hadi@kimia.fs.utm.my
}

Received 17 February 2008

http://dx.doi.org/10.11113/mjfas.v4n1.33

\section{ABSTRACT}

A novel method for synthesizing polyvinyl alcohol (PVA) embedded gold film is presented. Gold particles, in the size range of 20 to $180 \mathrm{~nm}$, were first prepared by the conventional Turkevitch method by the reduction of gold, hydrogen tetrachloroaurate $\left(\mathrm{HAuCl}_{4}\right)$ with sodium citrate in water. The resulting gold nanoparticles were characterized by ultra violet-visible (UV-Vis) absorption spectroscopy, dark-field microscopy, transmission electron microscopy (TEM) and field emission electron microscopy (FESEM). In the preparation of PVA embedded gold film, PVA was functionalized with (3-mercaptopropyl) trimethoxysilane (MPTMS) which produced a thiol functionality on the surface. Then, gold particles were embedded on the surface of partially dried functionalized PVA where the gold particles are chemisorbed onto the thiol groups. Their physical properties were studied using Fourier transform infra red spectroscopy (FTIR), FESEM, TEM and UV-Vis diffuse reflectance (UV-Vis DR). Considering that the gold nanoparticles in solution cannot possibly be recovered and reused, the PVA embedded gold film on the other hand, has potential to be reused multiple of times.

| Gold Nanoparticles | Polyvinyl Alcohol | Biosensor | Surface Plasmon Resonance |

\section{Introduction}

Nanotechnology is an anticipated manufacturing technology that allows thorough, inexpensive control of the structure of matter by working with atoms. It allows many things to be manufactured at low cost and with no pollution. Nanotechnologies can extend the long-established trend toward smaller, faster, cheaper materials and devices. Sometimes called the building block of nanotechnology, nanoparticles (particles with diameter less than $100 \mathrm{~nm}$ ) constitute a commercially important sector of the nanotechnology market. Recent intense interest in nanoparticles stems from the fact that, materials at the nanoscale exhibit unique optical, electronic and magnetic properties not seen in the bulk scale, which makes nanostructures attractive for a wide range of applications. In particular, nanostructures made from the noble metals, such as gold, with their associated strong surface plasmon resonance (SPR) have attracted considerable attention.

Colloidal gold have been used technologically for a very long time now. In the $17^{\text {th }}$ century, the brilliant colours of nanosized colloidal particles of $\mathrm{Ag}, \mathrm{Au}$ and $\mathrm{Cu}$ were used in staining glasses [1]. Gold nanoparticles scatter light intensely and are much brighter than chemical fluorophores. They have ready bioconjugation, potential 
noncytotoxicity, not susceptible to photobleaching and excellent biocompatibility to biomolecules. Furthermore, gold nanoparticles can easily be detected in as low as $10^{-16} \mathrm{M}$ concentration and have strong binding affinity towards thiols, disulfides and amines [2].

The brilliant colours exhibited by gold nanoparticles in the visible and near-infrared spectral regions can be attributed to their fascinating SPR properties. SPR is an optical phenomenon arising from the interaction between an electromagnetic wave and the conduction electrons in a metal. Under the irradiation of light, the conduction of electrons in a gold nanostructure are driven by the electric field to collectively oscillate at a resonant frequency relative to the lattice of the positive ions. At this resonant frequency, the incident light is absorbed by the nanostructure. Some of the photons will be released in all direction and this process is known as scattering. At the same time, some of these photons will be converted into phonons or vibrations of the lattice and this process is referred to as absorption. In general, the SPR peak of a gold nanostructure consists of two components; scattering and absorption [3]. The SPR spectrum depends on the nanoparticles itself (i.e. its size, shape and material) but also on the external properties of the nanoparticles environment. This makes noble metal nanoparticles extremely valuable from sensing point of view. Additionally, gold nanoparticles are promising candidates for applications in nanodevices, nanoelectronics, biolabeling biosensors.

Recent studies have shown that gold nanoparticles have immense potential for cancer diagnosis and therapy on account of their SPR enhanced light scattering and absorption [4]. Gold bioconjugates have been used for vital imaging of precancerous and cancerous cells by researches for in vitro and in vivo experiments [5]. Other than that, gold nanoparticles efficiently convert the strongly absorbed light into localized heat, which can be exploited for selective laser photothermal therapy of cancer. So far, the results are extremely promising. What makes this approach so promising is that it does not require expensive high powered microscopes or lasers to view the results, as other technique require. All it takes is a simple, inexpensive optical microscope and white light [2,4].

However, the method used was not practical because in the above method, the gold nanoparticles were in solution form such that it cannot be recovered and reused. Furthermore, most nano-sized metal are very unstable. They can aggregate because of the high surface free energy and can be oxidized-contaminated by air, moisture, $\mathrm{SO}_{2}$ and so on. Therefore, the embedding of nanoscopic metals into polymer represents a valid solution to the manipulation and stabilization problems.

In this research, novel functionalized gold nanoparticles embedded on the surface of polymer will be prepared for the application of biological diagnostics whereby the results can be viewed simply by employing an optical microscope and white light.

\section{Materials and Methods}

\section{Synthesis and Characterization}

All the chemicals were used as received without further purification. UV-Vis studies of gold nanoparticles solutions were carried out using PerkinElmer Lambda $25 \mathrm{UV}-\mathrm{Vis}$ spectrophotometer, using a $1 \mathrm{~cm}$ quartz cell. The single absorbance peak in the range of $520-550 \mathrm{~nm}$ is due to the characteristic surface plasmon absorption band of the gold nanoparticles. Optical imaging of gold nanoparticles was characterized using a 75W Xenon light source and CXR3 Trinocular Research Microscope (Labomed) in epi-illuminated darkfield mode. Images were collected through a 40x, 0.5 NA darkfield objective and detected using a CCD camera. The surface morphology of the samples was conducted using Jeol JSM-6701F FESEM operating from $2.0 \mathrm{kV}$ to $5.0 \mathrm{kV}$. TEM micrographs were taken with a Philip CM12 electron microscope (Philips Electron Optics, Eindhoven, Netherlands) operating at $80 \mathrm{kV}$. Infrared spectra were performed on a Nicolet Avatar-370 DTGS instrument 
(Thermo Electron Corporation) in the $4000-650 \mathrm{~cm}^{-1}$ range. UV-Vis Diffuse Reflectance (UV-Vis DR) of PVAGold film was recorded using Perkin Elmer Lambda 900 UV-VIS-NIR spectrometer.

\section{Synthesis of Gold Nanoparticles in Different Sizes and Shapes}

\section{Synthesis of $20 \mathrm{~nm}$ Gold Nanoparticles}

The $20 \mathrm{~nm}$ gold nanoparticles were synthesized by the citrate reduction of $\mathrm{HAuCl}_{4}$ in water. A $250 \mathrm{~mL}$ double neck round bottom flask was cleaned in aqua regia $\left(3 \mathrm{HCl}: 1 \mathrm{HNO}_{3}\right)$ and rinsed with distilled water. $100 \mathrm{~mL}$ of 1 $\mathrm{mM} \mathrm{HAuCl}$ solution $\left(0.04 \mathrm{~g} \mathrm{HAuCl} \mathrm{H}_{4} 3 \mathrm{H}_{2} \mathrm{O}\right.$ in $100 \mathrm{~mL}$ water) was heated to boiling, refluxed while being stirred (Figure 3.1$)$. Then, $10 \mathrm{~mL}$ of a $38.8 \mathrm{mM}$ sodium citrate solution $(0.1141 \mathrm{~g}$ sodium citrate in $10 \mathrm{~mL}$ water) is added quickly. The solution turned colour from yellow to black and to deep red. After the colour changed, the solution was refluxed for an additional 15 minutes. Then, the heater was turned off and the solution was stirred until it reached cool to room temperature.

\section{Synthesis of $30 \mathrm{~nm}$ to $180 \mathrm{~nm}$ Gold Nanoparticles}

$50 \mathrm{~mL}$ of $0.01 \% \mathrm{HAuCl}_{4}$ solution $(0.01 \mathrm{~g}$ in $100 \mathrm{~mL}$ water) was heated to boiling while being stirred in a $100 \mathrm{~mL}$ conical flask (Figure 3.2). Then a few hundred $\mu \mathrm{L}$ of $1 \%$ sodium citrate solution is quickly added to the auric solution. The solution changed colour within minutes from yellow to black and then to red or purple colour depending on the sizes of the nanoparticles. After the colour changed, the solution was stirred for an additional 10 minutes. The colour change for larger nanoparticles was slower compared to smaller nanoparticles. The amount of citrate solution determines the size of the nanoparticles synthesized. The approximate amount of citrate and the corresponding sizes of nanoparticles are listed in Table 1.

Table 1 : Approximate amount of citrate and the corresponding sizes of nanoparticles.

\begin{tabular}{cc}
\hline Citrate amount $(\mu \mathrm{L})$ & Nanoparticles size $(\mathrm{nm})$ \\
\hline 350 & 30 \\
330 & 40 \\
260 & 50 \\
230 & 60 \\
210 & 80 \\
\hline
\end{tabular}

\section{Synthesis of PVA Embedded Gold Film (PVA-Gold)}

PVA was dissolved in distilled water at $90^{\circ} \mathrm{C}$ for an hour to obtain $7 \mathrm{wt} \%$ PVA homogeneous solutions. The hot solution was filtered and then, $0.7 \mathrm{~g}$ of MPTMS and an appreciable amount of $1 \mathrm{M} \mathrm{HCl}$ was added to the filtrate to adjust the $\mathrm{pH}$ of the medium to 1.5 . After that, the mixture was stirred for 12 hour at room temperature. The PVA solutions were cast on a glass slide and dried at room temperature for 1 day and then the dried film were annealed at $100^{\circ} \mathrm{C}$ for an hour. Next, a few drops of gold nanoparticles solution were deposited on the surface of the dried PVA membrane and was annealed again for an hour. The resulting PVA membrane was peeled off from the glass slide for characterization. 


\section{Results and Discussion}

\section{Colour and UV-Vis Absorption Property of Different Sized Gold Nanoparticles}

The amount of citrate solution determines the size of the nanoparticles. Larger gold particles were produced when the amount of citrate were reduced [11]. When a high citrate amount is added, the negative citrate ions adsorbed onto the gold nanoparticles is increased and this introduce the surface charge that repels the particles and prevent them from agglomerating. However, when less citrate amount was added, the coverage of the citrate ions is incomplete and thus, causes the particles to aggregate into larger particles.

The formation-growth of gold clusters was monitored by UV-Vis spectroscopy, looking at the very intensive SPR absorption in the visible region characterizing nano-sized gold. Figure 2 shows the UV-Vis absorption spectra of samples S1-S6. The SPR peak of sample S1 was located at $527 \mathrm{~nm}$. As the particle size increased from sample S1 - S6, the absorption peak is moved to higher wavelengths (red-shift, from $527 \mathrm{~nm}-543 \mathrm{~nm}$ ) due to the aggregation of the gold nanoparticles.

As the size of the gold particles increases, the colour of the solution varies from deep red solution to purple as the as shown in Figure 3. The different colours of the gold nanoparticles solution are due to its SPR properties. Nanoparticles can experience SPR in the visible portion of the electromagnetic spectrum. This means that a certain portion of visible wavelengths will be absorbed, while another portion will reflected. The portion reflected will lend the material a certain color. Small nanoparticles absorb light in the blue-green portion of the spectrum $(400-500 \mathrm{~nm})$ while red light $(700 \mathrm{~nm})$ is reflected, yielding a deep red color. As particle size increases, the wavelength of surface plasmon resonance related absorption shifts to longer, redder wavelengths. This means that red light is now adsorbed, and bluer light is reflected, yielding particles with purple colour.

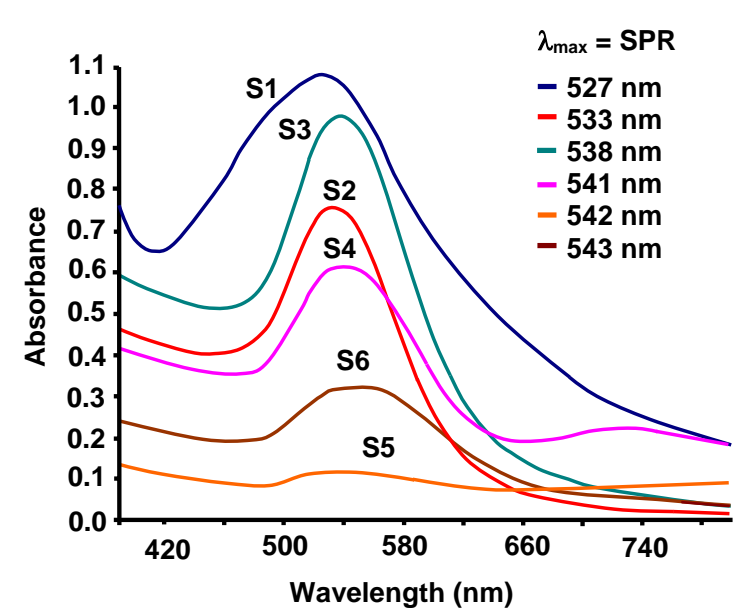

Figure 2: Absorption spectra of gold nanoparticle solutions for samples S1-S6.

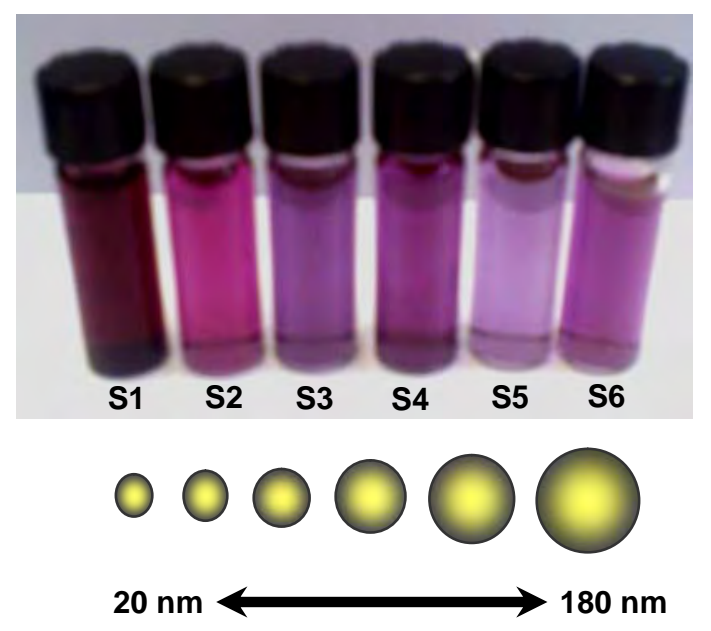

Figure 3: Photographs of colloidal dispersions of gold nanoparticles with increasing size for samples S1-S6.

\section{Surface Plasmon Scattering of Gold Nanoparticles}

Figure 4 shows a photograph of the gold nanoparticles observed under dark-field microscopy. Gold nanoparticles display a variety of plasmon resonance colours due to different size and shape of the particles. The blue particles 
(scattering between $400-480 \mathrm{~nm}$ ) are roughly spherical, green particles $(500-550 \mathrm{~nm})$ are typically hexagonal (larger diameter) and red particles $(600-700 \mathrm{~nm})$ have a triangular cross section, varying in thickness from platlets to tetrahedrons [12].

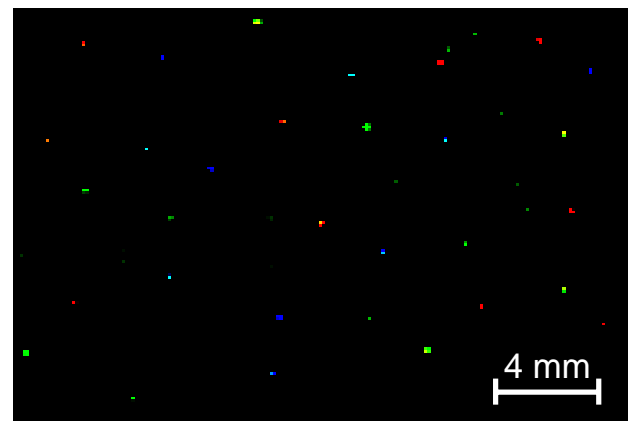

Figure 4: Photograph of gold nanoparticles viewed under optical microscope. The different colours correspond to different shape of the particles.

\section{Surface Morphology of Gold Nanoparticles}

Figure 5 illustrates FESEM micrographs of the synthesized gold nanoparticles with increasing diameters (images (a) - (f) for samples S1 - S6). The FESEM images show that the gold nanoparticles formed in all samples were in a homogeneous form. Other than that, it was observed that smaller sized particles are almost spherical in shape and as the size grew larger, the particles are more elongated.

The insets in Figures 5 (a) - (f) are corresponding TEM images of gold nanoparticles. TEM study of gold nanoparticles confirmed that the particles are almost spherical in shape with an average size ranging from 20 $180 \mathrm{~nm}$. From the TEM study, the average diameters for samples S1 - S6 were determined to be 27, 56, 83, 100, 102 and $116 \mathrm{~nm}$, respectively.
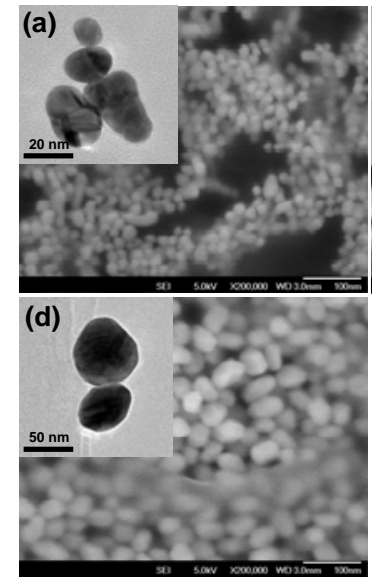
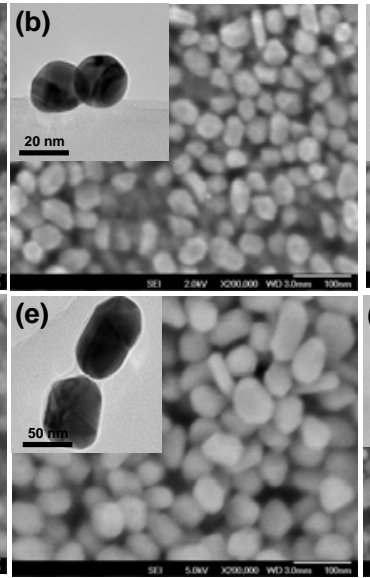
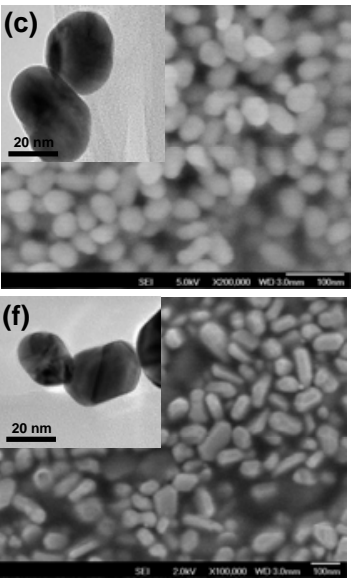

Figure 5 : FESEM images of gold nanoparticles prepared from different concentration of citrate. Images (a) - (f) correspond to samples S1 - S6. The inset in images (a) - (f) shows the TEM images of gold nanoparticles. 


\section{FTIR Spectra of PVA-Gold Film}

Figure 6 shows the FTIR spectra of PVA film, PVA-MPTMS film and PVA-Gold film in the range of 4000-750 $\mathrm{cm}^{-1}$. In all cases, there is a broad absorption noticed in the range of $3294-3370 \mathrm{~cm}^{-1}$. It is due to the $\mathrm{O}-\mathrm{H}$ stretching vibrations for the absorbed moisture and hydrogen bonded hydroxyl groups present in the sample. The absorption peak at $1000-1100 \mathrm{~cm}^{-1}$ is assigned to the stretching vibrations of $\mathrm{C}-\mathrm{O}$ and $\mathrm{C}-\mathrm{O}-\mathrm{C}$ groups in PVA (Figure 6(a)).

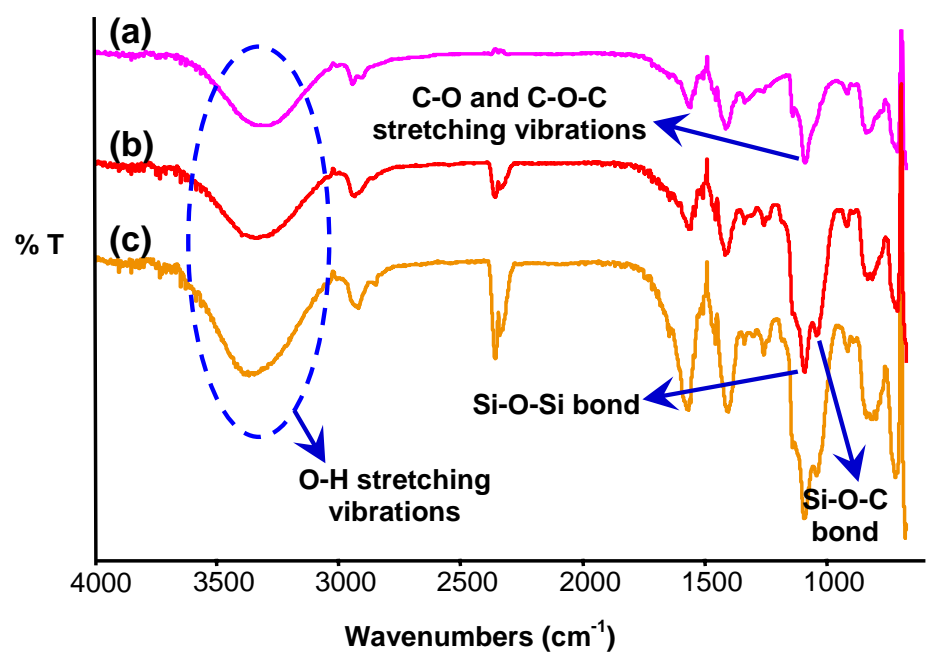

Figure 6 : FTIR spectra of (a) control PVA film, (b) PVA-MPTMS film prepared under acid condition and (c) PVA-Gold film.

An increase in the absorbance of the peak at $1000-1100 \mathrm{~cm}^{-1}$ of PVA-MPTMS film is attributed to the formation of $\mathrm{Si}-\mathrm{O}-\mathrm{C}\left(1043 \mathrm{~cm}^{-1}\right)$ and $\mathrm{Si}-\mathrm{O}-\mathrm{Si}\left(1093 \mathrm{~cm}^{-1}\right)$ bonds (Figure 6(b)). The $\mathrm{Si}-\mathrm{O}-\mathrm{Si}$ group is the result of condensation reaction between hydrolyzed silanol $\mathrm{Si}-\mathrm{OH}$ groups and the $\mathrm{Si}-\mathrm{O}-\mathrm{C}$ groups may be originated from the condensation reaction between $\mathrm{Si}-\mathrm{OH}$ and $\mathrm{C}-\mathrm{O}-\mathrm{H}$ groups from $\mathrm{PVA}$.

Therefore, the presence of $\mathrm{Si}-\mathrm{O}-\mathrm{C}$ and $\mathrm{Si}-\mathrm{O}-\mathrm{Si}$ bonds confirmed the existence of covalent linkage between the organic groups and the silica, which led to better compatibility and crosslinking network between organic and inorganic components.

The appearance of gold nanoparticles could not be detected from IR spectroscopy as no changes were observed in the absorption spectra of PVA-MPTMS and PVA-Gold film (Figure 6(c)). Other characterization technique such as FESEM and TEM were used to detect the appearance of gold nanoparticles on polymer film.

\section{FESEM Characterization of PVA-Gold Thin Film}

The FESEM observation of the PVA film, PVA-MPTMS film and PVA-Gold film are shown in Figure 7 (a), (b) and (c) respectively. Obvious contrast can be seen between all three PVA samples. The surface of the PVA is homogeneous and the silica phase did not form. However, silica particles can be found in the surface of PVAMPTMS due to the polycondensation of MPTMS leading to conglomeration in the polymer matrix. 

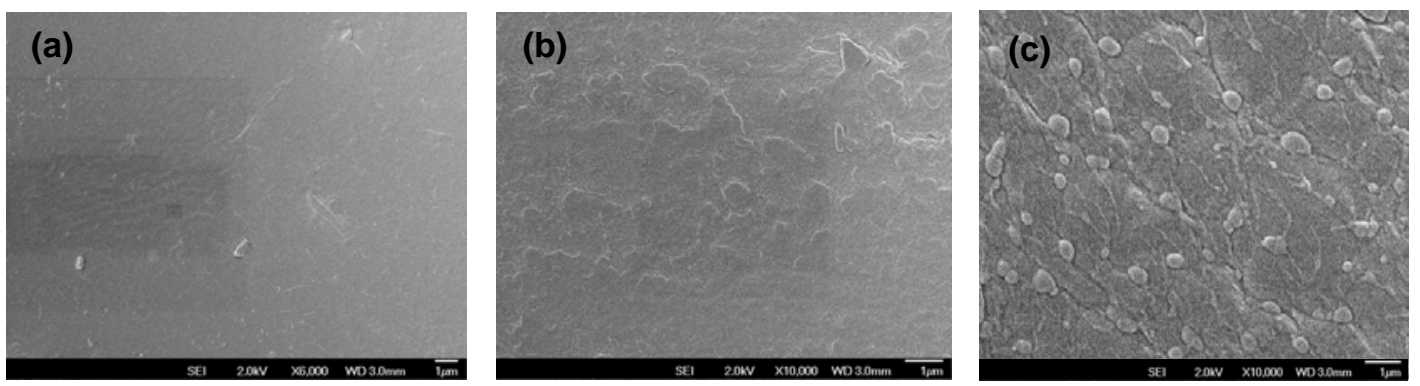

Figure 7 : FESEM micrograph of (a) PVA film, (b) PVA-MPTMS film and (c) PVA-Gold film.

\section{TEM Characterization of PVA-Gold Thin Film}

Figure 8 shows TEM micrograph of PVA-Gold film. The existence of gold nanoparticles on the surface of the polymer was evidenced by detection of dark spots. TEM micrograph of gold nanoparticles showed that the average size of the nanoparticles vary in between $30 \mathrm{~nm}-150 \mathrm{~nm}$.

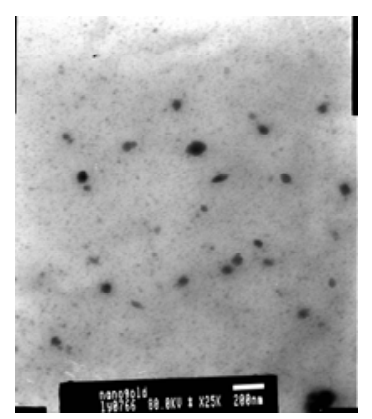

Figure 8 : TEM micrograph of PVA-Gold film.

UV-Vis Diffuse Reflectance (UV-Vis DR) of PVA-Gold Thin Film

Figure 9 displays UV-Vis DR spectrum of PVA-Gold film. The spectrum shows an absorbance peak at 527 $\mathrm{nm}$, which confirmed that gold nanoparticles are embedded on the surface of the polymer.

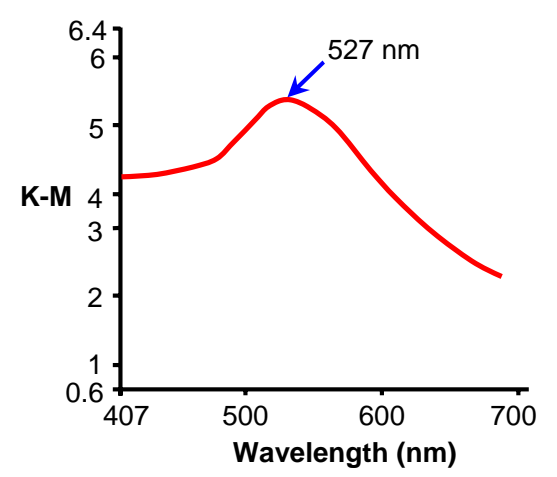

Figure 9 : UV-Vis DR spectrum of PVA-Gold film.

\section{Conclusion}

Gold nanoparticles embedded on the surface of PVA were successfully synthesized and characterized by FTIR, FESEM and TEM. Unlike the methods reported in previous literatures, the present method involves a facile fabrication route for the attachment of gold nanoparticles on the surface of PVA. Firstly, gold nanoparticles with diameters ranging from 20 to $180 \mathrm{~nm}$ were synthesized by the conventional Turkevitch method, which is the 
citrate reduction of gold salt in water. Then, the surface of PVA was functionalized with MPTMS which gives thiol tailored end-groups. Lastly, the gold nanoparticles were embedded on the functionalized PVA surface by annealing methods. This study is significant as the gold nanoparticles are not only bound to the thiol groups but also embedded in the polymeric layer which makes it a strong attachment. This study is also significant as it is also possible to incorporate other metal nanoparticles such as $\mathrm{Pd}, \mathrm{Pt}, \mathrm{Ag}$ and $\mathrm{Cu}$ having strong affinity for thiols.

\section{Acknowledgement}

This research was supported by the Ministry of Science, Technology and Innovation (MOSTI), Malaysia under Sciencefund Grant no. 03-01-06-SF0326 and the Ministry of Higher Education (MOHE), Malaysia under Fundamental Research Grant Scheme (FRGS) Vot. 78070.

\section{References}

[1] Jain, P. K., El-Sayed, I. H., El-Sayed, M. A. (2007). Au nanoparticles target cancer. Nanotoday. 2(1), 1829.

[2] El-Sayed, I. H., Huang, X., El-Sayed, M. A. (2005). Surface Plasmon Resonance Scattering and Absorption of anti-EGFR Antibody Conjugated Gold Nanoparticles in Cancer Diagnostics: Applications in Oral Cancer. Nano Letters. 5(5), 829-834.

[3] Hu, M., Chen, J., Li, Z., Au, L., Hartland, G. V., Li, X., Marquez, M., Xia, Y., (2006). Gold nanostructures: engineering their plasmonics properties for biomedical applications. Chem. Soc. Rev. 35.1084-1094.

[4] Huang, X., El-Sayed, I. H., Qian, W., El-Sayed, M. A. (2006). Cancer Cell Imaging and Photothermal Therapy in the Near-Infrared Region by using Gold Nanorods. J. Am. Chem. Soc. 128. 2115-2120.

[5] Durr, N. J., Larson, T., Smith, D. K., Korger, B. A., Sokolov, K., Ben-Yakar, A., (2007). Two-Photon Luminescence Imaging of Cancer Cells Using Molecularly Targeted Gold Nanorods. Nano Letters. 7(4). 941-945.

[6] Kimling, J., Maier, M., Okenve, B., Kotaidis, V., Ballot, H., Plech, A. (2006). Turkevitch Method for Gold Nanoparticles Synthesis Revisited. J. Phys. Chem. 110. 15700-15707.

[7] Zou, X., Ying, E., Dong, S. (2006). Seed-mediated synthesis of branched gold nanoparticles with the assistance of citrate and their surface-enhanced Raman scattering properties. Nanotechnology. 17(18). 4758-4764.

[8] Corbierre, M. K., Cameron, N. S., Sutton, M., Laaziri, K., and Lennox, R. B. (2005). Gold Nanoparticle/Polymer Nanocomposites: Dispersion of Nanoparticles as a Function of Capping Agent Molecular Weight and Grafting Density. Langmuir. 21(13). 6063 -6072.

[9] Bai, J., Li, Y., Yang, S., Du, J., Wang, S., Zheng, J., Wang, Y., Yang, Q., Chen, X. (2006). A simple and effective route for the preparation of poly(vinylalcohol) (PVA) nanofibers containing gold nanoparticles by electrospinning method. Solid State Communications. 141. 292-295.

[10] Tseng, J.-Y., Lin, M.-H., Chau, L.-K. (2001). Preparation of colloidal gold multilayers with 3(mercaptopropyl)-trimethylsilane as a linker molecule. Colloids and Surface A: Physichochemical and Engineering Aspects. 182. 239-245.

[11] Kimling, J., Maier, M., Okenve, B., Kotaidis, B., Ballot, H. and Plech, A. (2006). "Turkevitch Method for Gold Nanoparticles Synthesis Revisited". J. Phys. Chem. B. 110(32). 15700-15707.

[12] Gergo Szakmany (2005). "Optical Properties of Metal Nanoparticles”. University of Notre Dame, Notre Dame. 